\title{
Primary pleural hydatid cyst
}

\author{
Mallika Tewari · Vinay Kumar · Hari S. Shukla
}

Received: 15 September 2008 / Accepted: 19 September 2008

(C) Association of Surgeons of India 2009

Primary pleural hydatid cyst is an uncommon presentation of hydatid disease. We present one such patient with the cyst on the left lateral chest wall masquerading as chronic abscess.

A 28-year old lady presented with an enlarging, painless lump in the left chest wall for the last 6 months. There was no history of fever or allergic episodes. She had history

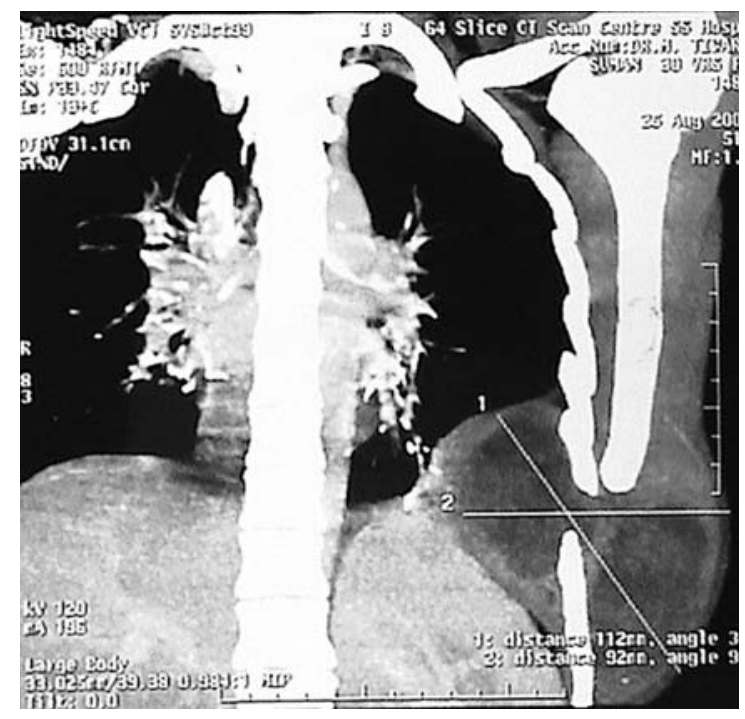

Fig. 1 Contrast-enhanced CT showing the extent of primary pleural hydatid cyst

M. Tewari $\cdot$ V. Kumar $\cdot$ H. S. Shukla

Department of Surgical Oncology

Institute of Medical Sciences,

Banaras Hindu University,

Varanasi - 221 005, India

M. Tewari $(\bowtie)$

E-mail: mallika_vns@satyam.net.in; drmtewari@gmail.com of aspiration of thick purulent fluid from the lesion twice outside. Examination revealed 10x8cm non-tender, cystic swelling between the left 7 th and the 8th ribs with round borders and the medial border was not palpable. Cough impulse was positive. Chest X-ray showed a smooth walled homogenous opacity left lower lung overlying the left dome of the diaphragm. Her abdominal sonography was normal. CECT (Figs. 1, 2) showed the presence of a large $16 \times 10$ $\mathrm{cm}$ dumb-bell shaped cystic lesion left pleural space extending from. The cyst cavity showed presence of brood capsules indicating it to be a primary hydatid disease of the pleura. The cyst was excised and patient discharged on Albendazole.

It is important to keep hydatid disease as one of the rare but possible differential diagnosis in cystic lesions of the chest wall in this endemic zone. Blind aspiration should be condemned unless a correct otherwise diagnosis is reached.

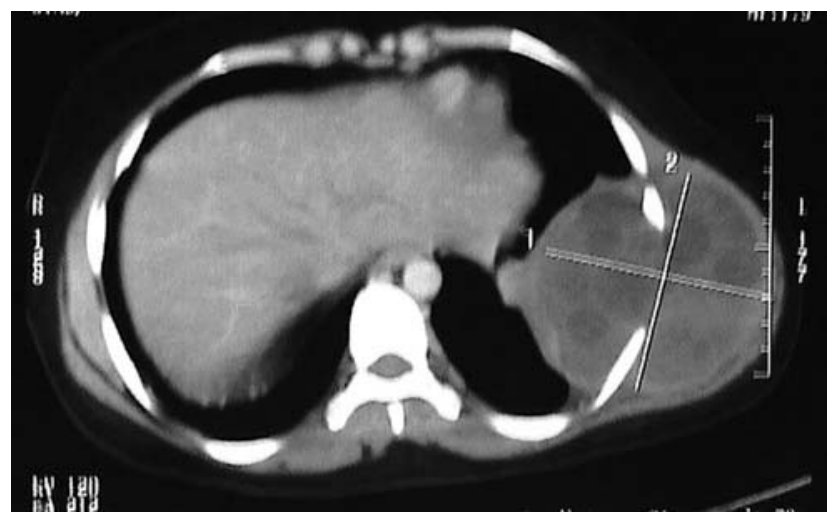

Fig. 2 Axial scan showing a large primary pleural hydatid cyst with brood capsules

Conflict of interest The authors do not have any disclosable interest 